\title{
Agrobacterium tumefaciens-mediated genetic transformation of the Taxol-producing endophytic fungus Ozonium sp EFY21
}

\author{
L. Liu ${ }^{1}$, Y.M. Wei ${ }^{1}$, X.W. Zhou' ${ }^{2}$, J. Lin ${ }^{1}$, X.F. Sun ${ }^{1}$ and K.X. Tang ${ }^{1,2}$ \\ ${ }^{1}$ State Key Laboratory of Genetic Engineering, School of Life Sciences, \\ Fudan University, Shanghai, China \\ ${ }^{2}$ Plant Biotechnology Research Center, School of Agriculture and Biology, \\ Shanghai Jiao Tong University, Shanghai, China
}

Corresponding author: K.X. Tang

E-mail: kxtang1@yahoo.com

Genet. Mol. Res. 12 (3): 2913-2922 (2013)

Received September 14, 2012

Accepted April 11, 2013

Published August 12, 2013

DOI http://dx.doi.org/10.4238/2013.August.12.7

\begin{abstract}
An efficient Agrobacterium tumefaciens-mediated genetic transformation method was successfully established for a newly isolated Taxol-producing fungus, Ozonium sp EFY21. A specific hygromycin B resistance expression vector, pCAMBIA1304'AN7-1, was constructed for fungal transformation. Key factors affecting transformation efficiency were thoroughly investigated and optimized. PCR amplification and Southern hybridization were used to verify the transformation events. This study should pave the way for future genetic modification studies of Ozonium sp EFY21.
\end{abstract}

Key words: Taxol-producing fungus; Protoplast; Genetic transformation; Agrobacterium tumefaciens 


\section{INTRODUCTION}

Taxol ${ }^{\circledR}$ is a well-known antitumor drug. Since Taxol was first marketed for the treatment of ovarian cancer in 1992, it has been used alone or in combination with other drugs for the treatment of a wide range of cancers (Suffness and Wall, 1995; Amikura et al., 2006; Kumar Naraharisetti et al., 2007; Fu et al., 2009; Oh et al., 2011). Taxol was discovered in the bark of Taxus brevifolia Nutt. (Wani et al., 1971) and for decades has been produced mainly by extraction from the bark and needles of yew trees. However, many factors, especially the dwindling numbers of Taxus trees and the extremely low Taxol concentration, severely limit its market supply. Due to continuously growing demand, many efforts have been made to find an economical and environmentally friendly way to produce Taxol. The first Taxol-producing fungus that was discovered, Taxomyces andreanae, was isolated from the bark of a yew tree (Stierle et al., 1993). Since then, many other Taxol-producing fungi have been reported (Raja et al., 2008; Kumaran et al., 2008, 2009; Geng et al., 2010; Kathiravan and Sri Raman, 2010), thereby opening avenues for the possibility of Taxol production by microbial fermentation.

Ozonium sp EFY21 is a Taxol-producing endophytic fungus isolated in our laboratory from wild Taxus chinensis var. mairei (Zhou et al., 2007). However, low yield precludes its practical use in industrial-scale production. To solve this problem, strain improvements as well as optimization of fermentation conditions are being exhaustively studied in our laboratory. Molecular biology techniques are powerful tools for strain improvement in the case of microorganisms. The establishment of an effective transformation system is a crucial prerequisite for applying molecular approaches for the improvement of Ozonium sp EFY21. A PEG (polyethylene glycol)-CaCl 2 -mediated transformation method for Ozonium sp EFY21 had been established in our laboratory, but the transformation efficiency is low and the transformants are unstable. An effective Ozonium sp EFY21 transformation system with high efficiency has yet to be established.

Agrobacterium tumefaciens-mediated genetic transformation (ATMT) was formerly used for plant genetic transformations. Since the time ATMT was used to transform Saccharomyces cerevisiae (Schiestl and Petes, 1991), many different types of fungi have been genetically transformed by this technique (Bundock et al., 1999; Aimi et al., 2005; Michielse et al., 2005; Betts et al., 2007). In the present study, ATMT was successfully applied to Ozonium sp EFY21 genetic transformation and key transformation parameters were evaluated. The optimized ATMT procedure dramatically enhances the transformation efficiency and should greatly facilitate the introduction of genetic modifications into Taxol-producing fungi for yield improvement studies.

\section{MATERIAL AND METHODS}

\section{Fungal culture}

Ozonium sp EFY21 was used as a recipient for ATMT and was cultivated in the dark at $28^{\circ} \mathrm{C}$ on YPS solid medium $\left(20 \mathrm{~g} / \mathrm{L}\right.$ sucrose, $10 \mathrm{~g} / \mathrm{L}$ tryptone, $5 \mathrm{~g} / \mathrm{L}$ yeast extract, $1 \mathrm{~g} / \mathrm{L} \mathrm{MgSO}_{4}$, $1 \mathrm{~g} / \mathrm{L} \mathrm{K}_{2} \mathrm{HPO}_{4}, 15 \mathrm{~g} / \mathrm{L}$ agar, $\left.\mathrm{pH} 6.8\right)$.

\section{Bacterial strains}

A. tumefaciens EHA105 was used as the host for maintenance of the transformation 
vector and for ATMT. It was cultured at $28^{\circ} \mathrm{C}$ on Luria-Bertani medium supplemented with $50 \mu \mathrm{g} / \mathrm{mL}$ rifampicin and $25 \mu \mathrm{g} / \mathrm{mL}$ streptomycin. Escherichia coli DH5 $\alpha$ was used for all molecular manipulations and was cultured on Luria-Bertani medium at $37^{\circ} \mathrm{C}$.

\section{Plasmid construction}

pCAMBIA1304 and pAN7-1 were the two starting plasmids used in this study. pCAMBIA1304 provided the skeleton for transfer DNA (T-DNA) and kanamycin selection in Agrobacterium, while pAN7-1 provided an expression cassette for hygromycin B selection in Ozonium sp EFY21. First, pCAMBIA1304 was cleaved with XhoI to remove the internal hygromycin B-resistance gene, and a 11.2-kb fragment was size selected, self-ligated, and introduced into E. coli DH5a, yielding pCAMBIA1304'. pCAMBIA1304' was digested with $B g l I I$ and HindIII and the large fragment was ligated with a 3.9-kb BglII-HindIII fragment from plasmid pAN7-1 containing a hygromycin B resistance expression cassette, yielding the target plasmid pCAMBIA1304'AN7-1 (Figure 1).

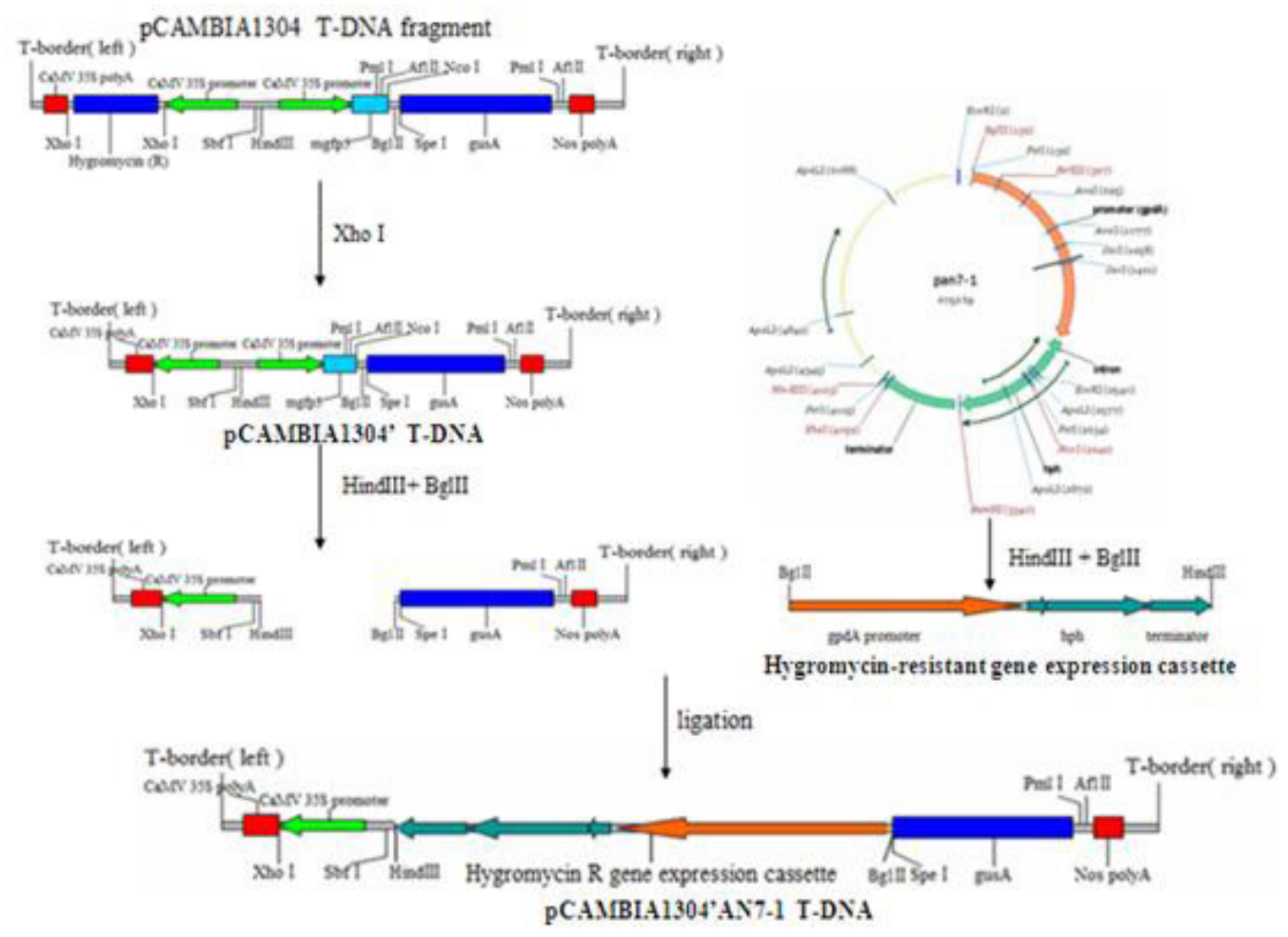

Figure 1. Construction of pCAMBIA1304'AN7-1 for fungal transformation.

\section{Protoplast preparation}

Protoplasts of Ozonium sp EFY21 were prepared as previously described (Wei et al., 
2010), with some modifications. Cultured EFY21 mycelia were collected by centrifugation and washed once with sterile distilled water, then once with $0.1 \mathrm{M}$ Tris-EDTA solution, and finally once with $0.7 \mathrm{M} \mathrm{NaCl}$ solution. Five percent (w/v) Lywallzyme ${ }^{\mathrm{TM}}$ solution was mixed with wet mycelia at a ratio of $1 \mathrm{~mL} / 250 \mathrm{mg}$ wet weight. The mixture was incubated in a rotary shaker at $30^{\circ} \mathrm{C}$ and $150 \mathrm{rpm}$ for $4-4.5 \mathrm{~h}$. After enzyme digestion, the crude mixture was filtered; it was first filtered through 4 layers, then 5, and finally through 6 layers of sterilized abrasive mirror paper. Subsequently, it was centrifuged at $4000 \mathrm{rpm}$ for $5 \mathrm{~min}$. The deposit was washed twice with STC buffer (1.2 M sorbitol, $25 \mathrm{mM} \mathrm{CaCl}_{2}$, and $0.01 \mathrm{M}$ Tris-HCl, $\mathrm{pH}$ 7.5) and resuspended in STC for later use.

\section{Genetic transformation of EFY21 protoplasts by ATMT}

The ATMT transformation protocol was carried out as previously described (de Groot et al., 1998), with some modifications. A. tumefaciens EHA105 harboring pCAMBIA1304'AN7-1 was cultured in $5 \mathrm{~mL}$ minimal medium (MM) (Hooykaas et al., 1979), supplemented with 50 $\mu \mathrm{g} / \mathrm{mL}$ rifampicin, $20 \mu \mathrm{g} / \mathrm{mL}$ streptomycin, and $75 \mu \mathrm{g} / \mathrm{mL}$ kanamycin at $250 \mathrm{rpm}$ and $28^{\circ} \mathrm{C}$ for $48 \mathrm{~h}$ until its $\mathrm{OD}_{600}$ value reached 1.5. The strain was collected by centrifugation and washed once with induction medium (IM) composed of MM with $40 \mathrm{mM}$ 2-(N-morpholino) ethanesulfonic acid, pH 5.3, $10 \mathrm{mM}$ glucose, and 0.5\% glycerol (w/v) (Bundock et al., 1995), and then resuspended in $\mathrm{IM}$; its $\mathrm{OD}_{600}$ value was adjusted to $0.15-0.20$. The bacterial suspension was further cultured until its $\mathrm{OD}_{600}$ value reached approximately 0.6. An aliquot of EFY21 protoplasts was mixed with an equal volume of activated $A$. tumefaciens EHA105 and the mixture was spread on a cellophane sheet that was placed on the cocultivation medium (CM) (IM medium with added agar) and cultured at $22-23^{\circ} \mathrm{C}$ for $48 \mathrm{~h}$. After cocultivation, the cellophane sheet was transferred to select medium (MM supplemented with $150 \mu \mathrm{g} / \mathrm{L}$ hygromycin $\mathrm{B}, 250 \mu \mathrm{g} / \mathrm{mL}$ carbenicillin, and $250 \mu \mathrm{g} / \mathrm{mL}$ cefotaxime and cultured at $28^{\circ} \mathrm{C}$ for several days until hygromycin B-resistant transformants appeared.

\section{Stability of hygromycin B-resistant transformants}

To evaluate the stability of hygromycin B-resistant transformants, randomly selected putative transformants were cultured on YPS solid medium for five generations in the absence of hygromycin B and then transferred to YPS solid medium containing $150 \mu \mathrm{g} / \mathrm{L}$ hygromycin B to determine mitotic stability.

\section{Fungal genomic DNA extraction, PCR verification, and Southern analysis}

Extraction of fungal genomic DNA was carried out as previously described (Sweigard et al., 1990). Confirmation of the presence of a T-DNA fragment was carried out by amplifying the hygromycin B resistance gene $(h p h)$ of pAN7-1. The PCR conditions were as follows: initial denaturation at $94^{\circ} \mathrm{C}$ for $5 \mathrm{~min}$ followed by 32 cycles of $94^{\circ} \mathrm{C}$ for $40 \mathrm{~s}, 56^{\circ} \mathrm{C}$ for $45 \mathrm{~s}$ and $72^{\circ} \mathrm{C}$ for 1 min, using the primers hphF: 5'-CACTGGCAAACTGTGATGGAC-3' and hphR: 5'-GCTG TATCTGGAAGAGGTAAACCC-3'. PCR products were detected on $1 \%$ agarose gels.

Southern blot hybridization was performed to determine the integration pattern of T-DNA. Procedures for genomic DNA digestion, agarose gel electrophoresis, and mem- 
brane transfer were those described by Sambrook and Russell (2001). Probe labeling, hybridization and signal detection were carried out based on the recommendations in Gene Image AlkPhos Direct Labeling and Detection System (Amersham, UK). Primers (HygS: 5'-AGGGCGAAGAATCTCGTGCTTTC-3', HygAS: 5'-ATGTTGGCGACCTCGTATTGGG A-3') designed to acquire the correct DNA fragment were used as probes and were based on the sequence of the $h p h$ gene of pAN7-1.

\section{RESULTS}

\section{Sensitivity of wild EFY21 to hygromycin B}

The antibiotic sensitivity of wild-type fungi was determined by culturing EFY21 on solid YPS medium supplemented with various concentrations of hygromycin B $(0,25,50$, $100,125$, and $150 \mu \mathrm{g} / \mathrm{L})$. The growth of wild-type EFY21 was progressively inhibited as the concentration of hygromycin B increased and was completely inhibited at $150 \mu \mathrm{g} / \mathrm{L}$ (Figure 2). Therefore, to ensure that all the transformants selected were authentic, $150 \mu \mathrm{g} / \mathrm{L}$ hygromycin B was used for selection.

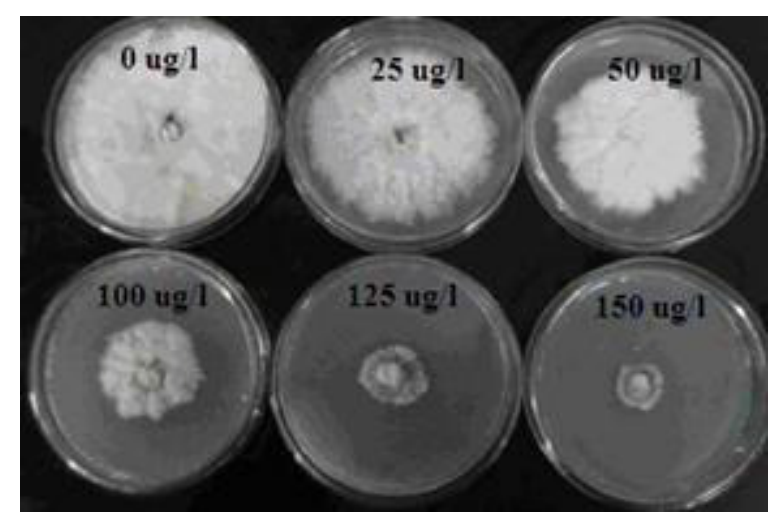

Figure 2. Growth inhibition assay for wild EFY21 cultured on YPS medium supplemented with different concentrations of hygromycin B $(0,25,50,100,125,150 \mu \mathrm{g} / \mathrm{L})$.

\section{Optimization of ATMT conditions for EFY21}

Cocultivation of EFY21 protoplasts with EHA105 harboring pCAMBIA1304'AN7-1 on $\mathrm{CM}$ for $48 \mathrm{~h}$ led to the formation of observable transformants about 3-4 days after the cellophane sheet was transferred to select medium (Figure 3).

Many factors were examined, including the use of different Agrobacterium strains, acetosyringone (AS) concentration and addition pattern, concentration of fungal protoplasts, and cocultivation conditions. Three different types of Agrobacterium strains (AGL-1, LBA4404, and EHA105) were tested, and the results showed that $A$. tumefaciens EHA105 was the most suitable for the ATMT of EFY21; few or no transformants were obtained when AGL1 and LBA4404 were used (Figure 4). As other studies have shown, successful fungal trans- 
formation with Agrobacterium requires the right bacterial strain. The first step for a particular fungal transformation by ATMT is therefore to identify an appropriate Agrobacterium strain.

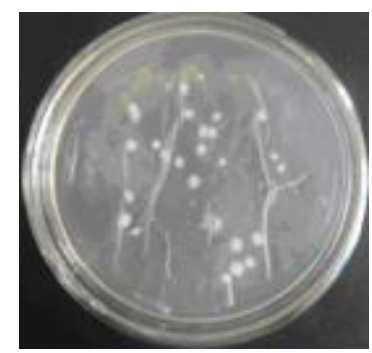

Figure 3. Result of Agrobacterium tumefaciens-mediated transformation for EFY21 protoplast.

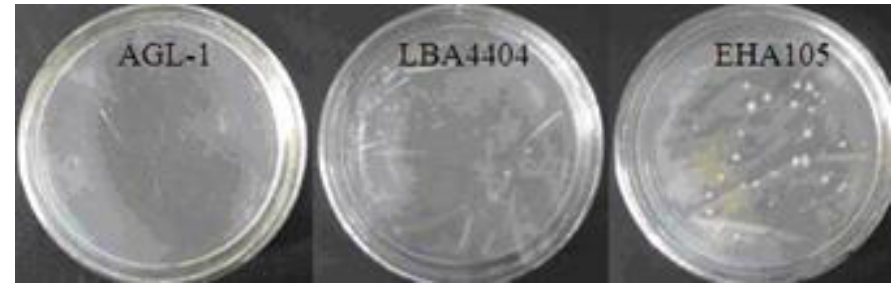

Figure 4. Effects of three different types of Agrobacterium strains on Agrobacterium tumefaciens-mediated transformation.

AS is another key factor in ATMT transformation. We used $200 \mu \mathrm{M}$, the same concentration as that used in many other studies. In this study, the addition pattern of AS was fully investigated. AS could be added at three different times: during Agrobacterium pre-culture, during Agrobacterium activation, and in the cocultivation period. The results showed that when AS was completely omitted, no transformants were observed. Addition of AS during the cocultivation period was absolutely necessary and was sufficient for obtaining many positive transformants. The addition of AS during the Agrobacterium culture cultivation period could increase transformation efficiency to some extent, but was not necessary (Figure 5).

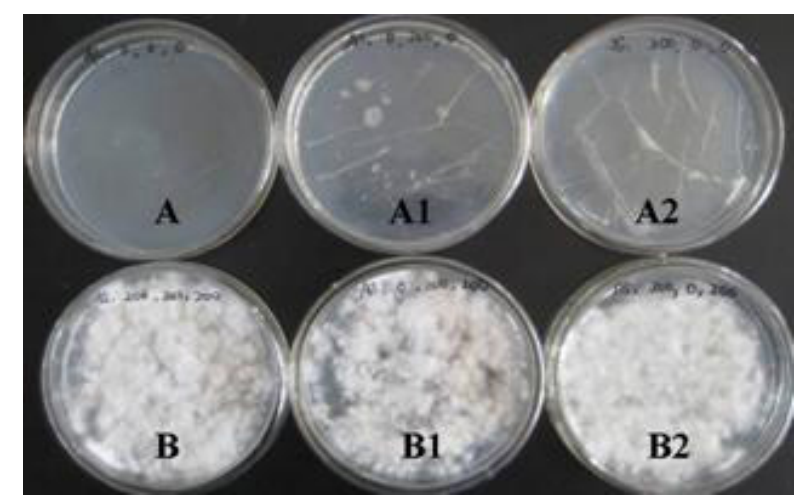

Figure 5. Transformation results by different addition patterns of acetosyringone in $\mu \mathrm{M}$ (A. 0, 0, 0; A1. 0, 200, 0; A2. 200, 0, 0; B. 200, 200, 200; B1. 0, 200, 200; B2. 200, 0, 200). 
The effect of protoplast concentration on ATMT was also examined. As anticipated, the number of transformants increased as the protoplast concentration increased (Figure 6). In this experiment, the Agrobacterium concentration was set at $\mathrm{OD}_{600} 0.6$, while the protoplasts were diluted to different concentrations. However, to obtain cleaner protoplasts, the filtration process was repeatedly operated so that the resultant protoplast concentration was always $10^{7} /$ $\mathrm{mL}$. The effect of Agrobacterium concentration was also studied. For OD values between 0.6 and 1.0, no significant increase in transformation efficiency was observed; therefore, the bacterial concentration was set at $\mathrm{OD}_{600} 0.6$.

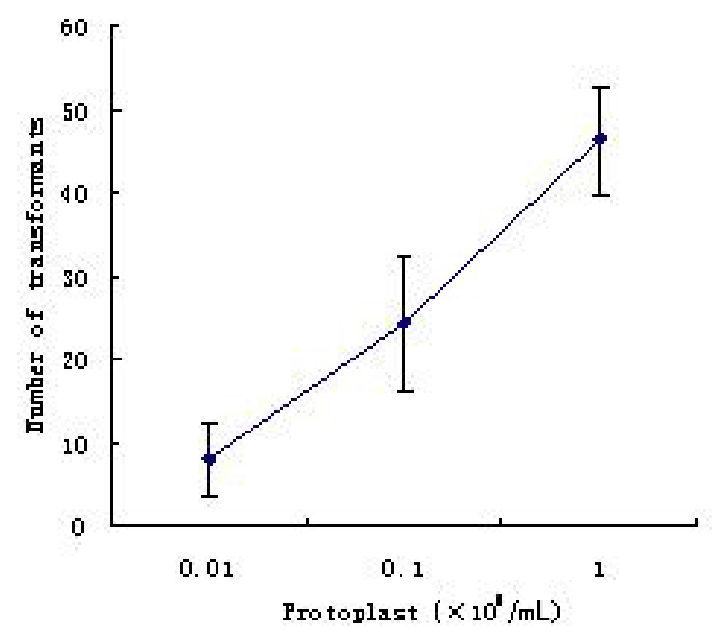

Figure 6. Effects of protoplast concentration on transformation efficiency.

According to our research, cocultivation conditions markedly affected transformation efficiency. In this experiment, different temperature and time combinations were studied. Very few transformants were formed when the cocultivation time was less than $24 \mathrm{~h}$, and the transformation efficiency significantly increased when the cocultivation time was longer than $36 \mathrm{~h}$. The highest efficiency was obtained following cocultivation for $48 \mathrm{~h}$ (Figure 7). Because the EFY21 mycelium grows luxuriously when cultivated on CM medium for significantly longer than $48 \mathrm{~h}$ and this would not be convenient for selection of transformants, a cocultivation time of $48 \mathrm{~h}$ was appropriate for the ATMT of EFY21. The effect of different temperatures was also studied and the results showed that temperatures between $20^{\circ}$ and $25^{\circ} \mathrm{C}$ were most suitable for EFY21 transformation. In this experiment, A. tumefaciens EHA105 concentration was set at $\mathrm{OD}_{600} 0.6$, and the protoplast concentration was $1 \times 10^{7} / \mathrm{mL}$.

\section{Stability of hygromycin B-resistant transformants}

Randomly chosen transformants were repeatedly cultured on solid YPS medium in the absence of hygromycin B followed by transferring fungal hyphal tips to YPS medium supplemented with $150 \mu \mathrm{g} / \mathrm{L}$ hygromycin B. The results showed that all the transformants selected maintained hygromycin B resistance (Figure 8). 


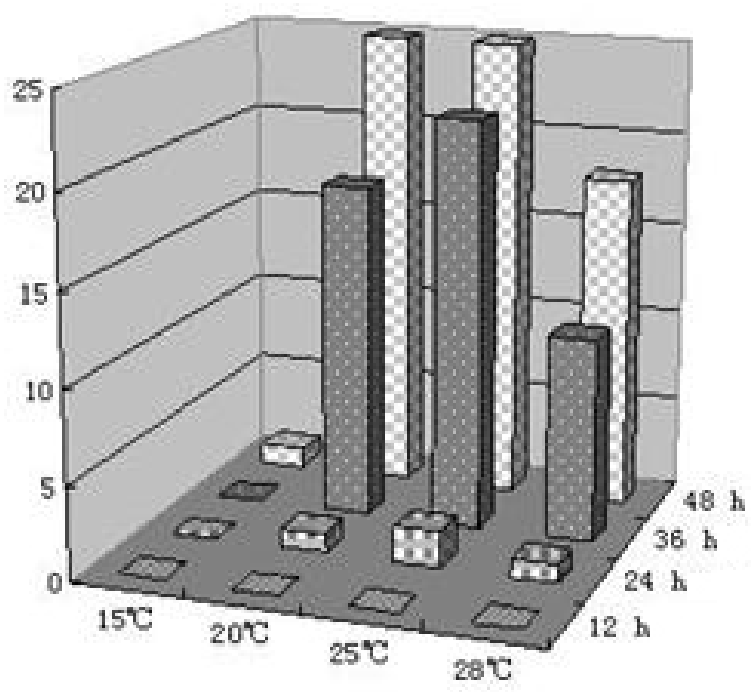

Figure 7. Effects of cocultivation conditions on transformation efficiency.

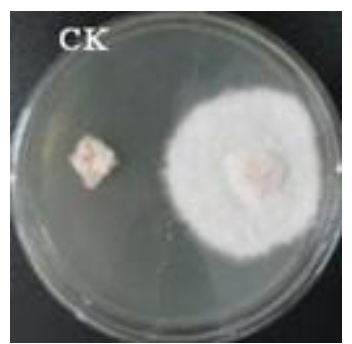

Figure 8. Stably inherited hygromycin B resistance of transformants. CK = wild-type EFY21.

\section{PCR verification and Southern analysis of transformants}

To confirm transformation events, PCR amplification was used to identify a specific $h p h$ gene fragment in the genomic DNA of transformants. The results (Figure 9) showed the presence of a 783-bp fragment of the $h p h$ gene in all the randomly selected transformants. Wild-type EFY21 was used as a negative control, and plasmid pAN7-1 was a positive control.

Southern hybridization was performed on transformants to analyze the integration mode of the T-DNA. Four transgenic fungal strains were randomly selected and analyzed along with wild-type EFY21 as a control. Genomic DNA was extracted and digested with $B g l I I$. The result showed that there was no hybridization signal with wild-type EFY21, while strong signals were observed with the hygromycin B-resistant fungal strains, demonstrating that T-DNA had inserted into the fungal genome with different numbers of copies (Figure 10). 


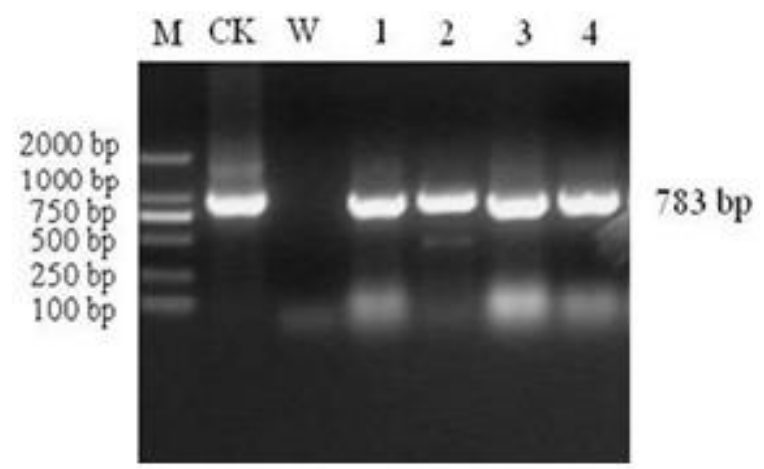

Figure 9. PCR verification of randomly selected hygromycin B-resistant transformants. Lane $M=$ DNA marker DL2000; lane $C K=$ pAN7-1 as positive control; lane $W=$ wild-type EFY21 as negative control; lanes 1-4 = randomly selected transformants.

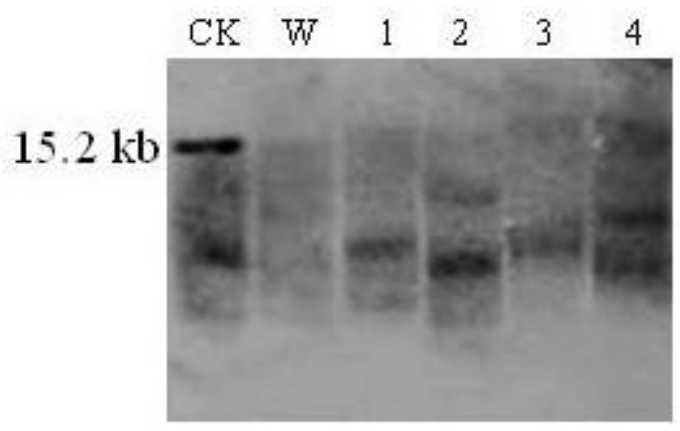

Figure 10. Southern blot analysis of transgenic fungal strains. Lane $C K=\mathrm{p}$ CAMBIA1304'AN7-1 as positive control; lane $W=$ wild-type EFY21 as negative control; lanes $1-4$ = randomly selected transformants.

\section{DISCUSSION}

Compared with traditional methods like PEG-mediated protoplast transformation, ATMT methods have many advantages, especially the high transformation efficiency and flexibility in fungal starting materials. In our study, both mycelium and protoplast were successfully used with the ATMT method. However, to gain enough transformants when space on Petri dishes is limited, EFY21 protoplasts were chosen for ATMT transformation.

In this study, an ATMT system with great stability and high efficiency was successfully established for the Taxol-producing endophytic fungus Ozonium sp EFY21. Several factors affecting transformation efficiency were tested and optimized. This study should facilitate strain improvement of EFY21 by molecular approaches and is a good reference for genetic study of other fungi.

\section{ACKNOWLEDGMENTS}

Research supported by the China National " 863 " High-Tech Program and the Shanghai Science and Technology Committee. 


\section{REFERENCES}

Aimi T, Taguchi H, Tanaka Y, Kitamoto Y, et al. (2005). Agrobacterium tumefaciens-mediated genetic transformation of the white root rot ascomycete Rosellinia necatrix. Mycoscience 46: 27-31.

Amikura T, Aoki Y, Banzai C, Yokoo T, et al. (2006). Metastatic choriocarcinoma successfully treated with paclitaxel and carboplatin after interstitial lung disease induced by EMA-CO. Gynecol. Oncol. 102: 573-575.

Betts MF, Tucker SL, Galadima N, Meng Y, et al. (2007). Development of a high throughput transformation system for insertional mutagenesis in Magnaporthe oryzae. Fungal Genet. Biol. 44: 1035-1049.

Bundock P, den Dulk-Ras A, Beijersbergen A and Hooykaas PJ (1995). Trans-kingdom T-DNA transfer from Agrobacterium tumefaciens to Saccharomyces cerevisiae. EMBO J. 14: 3206-3214.

Bundock P, Mróczek K, Winkler AA, Steensma HY, et al. (1999). T-DNA from Agrobacterium tumefaciens as an efficient tool for gene targeting in Kluyveromyces lactis. Mol. Gen. Genet. 261: 115-121.

de Groot MJ, Bundock P, Hooykaas PJ and Beijersbergen AG (1998). Agrobacterium tumefaciens-mediated transformation of filamentous fungi. Nat. Biotechnol. 16: 839-842.

Fu Y, Li S, Zu Y, Yang G, et al. (2009). Medicinal chemistry of paclitaxel and its analogues. Curr. Med. Chem. 16: 3966-3985.

Geng Z, Liu KH, Zhao YX, Li JH, et al. (2010). Isolation, identification of an endophytic taxol-producing fungus obtained from Taxus chinensis. Microbiol. China 37: 199-203.

Hooykaas PJJ, Roobol C and Schilperoort RA (1979). Regulation of the transfer of Ti-plasmids of Agrobacterium tumefaciens. J. Gen. Microbiol. 110: 99-109.

Kathiravan G and Sri Raman, V (2010). In vitro TAXOL production, by Pestalotiopsis breviseta - a first report. Fitoterapia 81: 557-564.

Kumar Naraharisetti P, Yung Sheng Ong B, Wei Xie J, Kam Yiu Lee T, et al. (2007). In vivo performance of implantable biodegradable preparations delivering Paclitaxel and Etanidazole for the treatment of glioma. Biomaterials 28: 886-894.

Kumaran RS, Muthumary J, Kim EK and Hur BK (2009). Production of taxol from Phyllosticta dioscoreae, a leaf spot fungus isolated from Hibiscus rosa-sinensis. Biotechnol. Bioprocess Eng. 14: 76-83.

Kumaran RS, Muthumary J and Hur BK (2008). Taxol from Phyllosticta citricarpa, a leaf spot fungus of the angiosperm Citrus medica. J. Biosci. Bioeng. 106: 103-106.

Michielse CB, Arentshorst M, Ram AF and van den Hondel CA (2005). Agrobacterium-mediated transformation leads to improved gene replacement efficiency in Aspergillus awamori. Fungal Genet. Biol. 42: 9-19.

Oh HC, Seo DW, Song TJ, Moon SH, et al. (2011). Endoscopic ultrasonography-guided ethanol lavage with paclitaxel injection treats patients with pancreatic cysts. Gastroenterology 140: 172-179.

Raja V, Kamalraj S and Muthumary JP (2008). Taxol from Botryodiplodia theobromae (BT115) - An endophytic fungus of Taxus baccata. J. Biotechnol. 136: 187-197.

Sambrook J and Russell DW (2001). Molecular Cloning: A Laboratory Manual. 3rd edn. Laboratory Press, Cold Spring Harbor.

Schiestl RH and Petes TD (1991). Integration of DNA fragments by illegitimate recombination in Saccharomyces cerevisiae. Proc. Natl. Acad. Sci. U. S. A. 88: 7585-7589.

Stierle A, Strobel G and Stierle D (1993). Taxol and taxane production by Taxomyces andreanae, an endophytic fungus of Pacific yew. Science 260: 214-216.

Suffness M and Wall ME (1995). Discovery and Development of Taxol. In: Taxol: Science and Applications. (Suffness M, ed.). CRC Press, Boca Raton. 3-25.

Sweigard JA, Orbach MJ, Valent B and Chumley FG (1990). A miniprep procedure for isolating genomic DNA from Magnaporthe grisea. Fungal Genet. Newsl. 37: 44.

Wani MC, Taylor HL, Wall ME, Coggon P, et al. (1971). Plant antitumor agents. VI. The isolation and structure of taxol, a novel antileukemic and antitumor agent from Taxus brevifolia. J. Am. Chem. Soc. 93: 2325-2327.

Wei Y, Zhou X, Liu L, Lu J, et al. (2010). An efficient transformation system of taxol-producing endophytic fungus EFY21 (Ozonium sp). Afr. J. Biotechnol. 9: 1726-1733.

Zhou X, Wang Z, Jiang K, Wei Y, et al. (2007). Screening of taxol-producing endophytic fungi from Taxus chinensis var. mairei. Appl. Biochem. Microbiol. 43: 439-443. 